\title{
Prescribing practices for malaria in a rural Ugandan hospital: evaluation of a new malaria treatment policy
}

\author{
Ucakacon PS ${ }^{1}$, Achan $\mathrm{J}^{2}$, Kutyabami $\mathrm{P}^{1}$, Odoi $\mathrm{AR}^{1}$, ${ }^{*}$ Kalyango $\mathrm{NJ}^{1,3}$ \\ 1. Department of Pharmacy, College of Health Sciences, Makerere University \\ 2. Department of Pediatrics and Child Health, College of Health Sciences, Makerere University \\ 3. Clinical Epidemiology Unit, College of Health Sciences, Makerere University
}

\begin{abstract}
Background: Increasing resistance to first line antimalarial drugs led to a change in the antimalarial policy of Uganda in 2005. Successful implementation of this policy depends on changing prescribing patterns of health workers.

Objectives: To describe prescribing patterns for malaria and associated factors in a rural Ugandan hospital following a change in antimalarial treatment policy from chloroquine plus sulphadoxine-pyrimethamine to artemisinin-based combination therapies.

Methods: From the outpatients register, 715 prescriptions for malaria were reviewed. Data was collected on patient demographics, prescriber factors and prescription patterns. Prescriptions were considered to conform to the new antimalarial policy if artemether-lumefantrine was prescribed for uncomplicated malaria or quinine for treatment failure or complicated malaria.

Results: The most frequently prescribed antimalarials for uncomplicated and complicated malaria were artemetherlumefantrine $(n=564,88.5 \%)$ and quinine $(n=66,84.6 \%)$ respectively. The proportion of prescriptions conforming to the new antimalarial treatment policy was $88.1 \%(\mathrm{n}=630)$. Predictors of conformity were: duration in service of less than 6 years $(\mathrm{OR}=3.40 . \mathrm{CI}=1.24-9.33)$, prescriber's profession $(\mathrm{OR}=97.51, \mathrm{CI}=27.29-348.34)$ and diagnosis of uncomplicated malaria (OR=10.13, CI=3.37-30.42).

Conclusions: Adequate training and provision of information relevant to the needs of different cadres of health workers is needed to promote effective uptake of new treatment policies.
\end{abstract}

Key words: Malaria policy, prescribing practices, malaria treatment

African Health Sciences 2011; 11(S1): S53 - S59

\section{Introduction}

Malaria remains an important public health concern, especially in tropical countries where almost all the infections occur. It is an important cause of death and illness in children and adults in tropical countries ${ }^{1}$. In Uganda, it is also the leading cause of illness and death, accounting for $25-40 \%$ of all outpatient visits at health care facilities, $20 \%$ of hospital admissions and $15 \%$ of inpatient deaths. Fighting the disease is therefore one of the highest priorities of the government of Uganda ${ }^{2}$.

Over the years there has been increasing spread of malaria parasites resistant to the commonly used antimalarial drugs ${ }^{3-5}$. In Uganda recent surveillance of antimalarial drug resistance

\begin{tabular}{|l|}
\hline *Corresponding author \\
Joan N Kalyango \\
College of Health Sciences \\
Makerere University \\
P.O Box 7072 \\
Kampala, Uganda \\
E mail: nakayaga2001@yahoo.com \\
\hline
\end{tabular}

African Health Sciences Vol 11 Special Issue 1 August 2011 showed that resistance to chloroquine and sulfadoxine-pyrimethamine in the treatment of uncomplicated malaria was widespread and had reached critical levels in most areas of the country ${ }^{6}$. As a result, the first line treatment for uncomplicated malaria was changed to Artemisinin-based combination therapy (ACTs), with the recommended second-line therapy remaining oral quinine. The recommended ACT in Uganda is artemetherlumefantrine $(\mathrm{AL})$ with the alternative of amodiaquine/artesunate (AQ/AS). Quinine remained the recommended treatment for complicated malaria ${ }^{7}$.

It is important, however, to realize that drug policy change and implementation for malaria case management in African settings is complex. It has been reported that a change in national therapeutic recommendations does not always translate to an immediate, effective policy change at health service provider level and does not necessarily translate into adequate quality case-management of patients at the point of care ${ }^{8}$. As such, the delivery of these drugs 
to the people who actually need them, as well as changing health practices actually remain a major challenge. Factors that influence implementation of policy guidelines are varied and include health worker and health facility factors ${ }^{9}$. Prescriber's adherence to treatment guidelines remains critical to the success of any new drug policy ${ }^{10}$. If prescribers do not adopt the new guidelines in the wake of widespread resistance to previously used antimalarial treatment regimens, malaria will continue to devastate the countries whose health systems are already overburdened by the communicable diseases. Performance of health workers could be evaluated by assessing their prescribing habits. Prescribing habits may be influenced by a number of factors including lack of training, shortage of drugs, financial influences, patient load, lack of materials and prescribing attitudes ${ }^{11}$, all of which are challenges in a typical health centre setting in Africa. There is limited information on the prescription practices of health workers after implementation of the 2005 antimalarial policy in Uganda. Some of the studies that have been done have focused mainly on the prescription of Artemether-Lumefantrine ${ }^{12}$ while others have looked at the management of malaria with the aid of rapid diagnostic tests ${ }^{13}$. In addition, factors associated with the prescription practices have not been well studied.

This study sought to describe the antimalarial prescribing habits of health care providers in a rural Ugandan hospital following implementation of a new antimalarial treatment policy and to determine the factors associated with these prescribing habits.

\section{Methods}

This was a cross-sectional study that involved a review of outpatient department prescriptions of 715 patients with a diagnosis of malaria during the months of October 2006 to January 2007.

The study setting was Kalisizo hospital in Rakai district, an area of medium to high malaria endemicity in the southern part of Uganda. This is a public hospital with a 100-bed capacity that offers free medical services and serves a population of about 300,000 people. It serves an average of 100 patients per day and about two thirds of these are malaria cases. The malaria burden in this area remains high despite the widespread use of interventions like Insecticide Treated Nets (ITNs) and provision of effective antimalarial drugs. The health workers in the hospital had all received training on the new antimalarial treatment policy. Kalisizo hospital was selected purposively to represent rural health facilities whose health workers had received training on the new malaria policy.

In addition, a rural setting was chosen because a large proportion of Ugandans (85\%) live in rural settings ${ }^{14}$. Thus, it was important to get results that would apply to this population. Systematic random sampling was used and the prescriptions for every third patient recorded with a diagnosis of malaria were reviewed. Prescriptions with missing information on key variables of interest were omitted. If a prescription was omitted, the next prescription that met the criteria was selected and thereafter the sequence of every third prescription was continued. The total number of records for malaria cases was 3,012 and we excluded 100 prescriptions from the review due to missing information. Sampling was done until a total sample size of 715 prescriptions was obtained. This sample size was estimated using a formula for estimation of a single proportion with adjustment for the expected clustering due to having prescriptions from the same prescribers ${ }^{15}$. The parameters used in the sample size estimation were: $95 \%$ confidence level, $5 \%$ level of precision, design effect of 1.8 and proportion of inappropriate prescriptions of $45 \%{ }^{16}$.

This computation gave a sample size of 685 , to which 30 prescriptions were added to cater for any missing characteristics. Data about patient demographics, prescriber factors and prescription patterns were collected by two trained research assistants using a pre-tested data extraction form. The research assistants were nurses working in Kalisizo hospital and were trained in data collection for this study for one week. Availability of drugs was assessed by looking at the stock cards for antimalarials and determining whether antimalarial drugs (artemether-lumefantrine and quinine) were in stock or not during the specific period when each of the patients was seen. Prescriptions were considered to conform to the 2005 antimalarial policy if artemether- lumefantrine was prescribed for uncomplicated malaria or if quinine was prescribed for treatment failure or complicated malaria. These classifications of malaria for the different patients were obtained from the patient register. The coding of whether treatment conformed to the new policy or not was done by the principal investigator based on the new policy treatment guidelines for complicated and uncomplicated malaria. We were not able to assess for adequacy of the dose prescribed due to insufficient information from the register. We 
were also not able to assess whether pregnancy or any other contra-indications had been ruled out because the records were not very comprehensive.

Data was double entered into Microsoft Access (Microsoft Corporation, Redmond, Washington), cleaned, coded and exported to STATA 8.0 (Stata, College Station, Texas, USA) for statistical analysis. Descriptive statistics were used for general description of the data and for description of prescribing patterns. Prescriptions were labeled as appropriate or inappropriate depending on whether they conformed to the 2005 antimalarial treatment policy. To determine the factors associated with conformity to the policy, generalized estimating equations with robust standard errors were used. The exchangeable correlation structure was assumed. This procedure uses generalized estimating equations to account for the potential correlation of treatment for patients seen by the same prescriber. The exchangeable correlation structure assumes the correlation of the treatment between any pair of patients is approximately constant.

Odds ratios and their 95\% confidence intervals were obtained using the binomial family and logit link. P-values less than 0.05 were considered statistically significant. Factors that had p-values less than 0.2 were considered for multivariate analysis. This was done to determine the independent predictors of non-conformity to the 2005 antimalarial policy, as well as confounders and effect modifiers. Age, sex, malaria diagnosis, duration in service and level of training were put in the multivariate model. Factors were considered as confounders if omitting them from the model caused a change in the magnitude of odds ratios of other factors of more than $10 \%{ }^{17}$.

The study was approved by the Department of Pharmacy of Makerere University, Kalisizo hospital administration and Faculty of Medicine Research and Ethics committee.

\section{Results}

We reviewed a total of 715 records. There were 9 prescribers who included 2 doctors, 5 clinical officers and 2 nurses, with median duration of service of 6 years (range 2 -14years). The clinical officers are health care professionals who provide advanced advisory, diagnostic, curative and preventive medical services. They undergo a three year medical training programme and can perform $60-80 \%$ of doctors' tasks. There were 169 (23.6\%) prescriptions from doctors, 488 from clinical officers $(68.3 \%)$ and 58 from nurses (8.1\%). About two thirds of the prescriptions were for female patients $(65 \%, \mathrm{n}=$ 465) and the median age of patients was 20 years (range 4 months - 94 years). About one third of the records were for children 12 years and below (34.7\%, $\mathrm{n}=248)$. Only 9.1\% ( $\mathrm{n}=65)$ of the patients had their weights recorded and only two of these were 12 years or less. Results of patients' demographics are summarized in Table 1.

Table 1: Patient characteristics

\begin{tabular}{lcc}
\hline Variable & Frequency & Percentage \\
Sex & 465 & 65.0 \\
Females & 250 & 35.0 \\
Males & \\
Age (median, min \& max) & \\
20 years & (4 months, 94 years) \\
Age & 248 & 34.7 \\
$\leq 12$ years & 466 & 65.3 \\
$>12$ years & & \\
Malaria diagnosis & 637 & 89.1 \\
Uncomplicated & 78 & 10.9 \\
\hline Complicated &
\end{tabular}

The majority of the prescriptions that were reviewed were for patients with uncomplicated malaria $(89.1 \%, \mathrm{n}=637)$. Artemether-Lumefantrine was prescribed for $88.5 \%(\mathrm{n}=564)$ of patients with uncomplicated malaria. Quinine was the most prescribed antimalarial for patients with complicated malaria $(84.6 \%, \mathrm{n}=66)$. SulphadoxinePyrimethamine (SP) alone, Chloroquine (CQ) alone and Chloroquine + Sulphadoxine-Pyrimethamine $(\mathrm{CQ}+\mathrm{SP})$ were prescribed mainly for uncomplicated malaria. A combination of AL + Quinine was also prescribed for 11 patients (1.5\%). Results for antimalarial prescription are summarized in Table 2. Prescribers conformed to the 2005 antimalarial treatment policy in $88.1 \%(n=630)$ of the prescriptions. 
Table 2: Antimalarials prescribed according to malaria classification

\begin{tabular}{llll}
\hline Antimalarial drug prescribed & $\begin{array}{l}\text { Uncomplicated } \\
\text { Malaria n (\%) }\end{array}$ & $\begin{array}{l}\text { Complicated } \\
\text { Malaria n } \mathbf{~ ( \% ) ~}\end{array}$ & Total N (\%) \\
\hline $\mathrm{AL}^{*}$ & $564(88.5)$ & $5(6.4)$ & $569(79.6)$ \\
Quinine & $8(1.3)$ & $66(84.6)$ & $74(10.4)$ \\
Chloroquine $+\mathrm{SP}^{\wedge}$ & $26(4.1)$ & $0(0.0)$ & $26(3.6)$ \\
$\mathrm{SP}^{\wedge}$ & $15(2.4)$ & $2(2.6)$ & $17(2.4)$ \\
Chloroquine & $15(2.4)$ & $0(0.0)$ & $15(2.1)$ \\
Quinine + AL* & $8(1.3)$ & $3(3.9)$ & $11(1.5)$ \\
$\mathrm{AL}^{*}+\mathrm{SP}^{\wedge}$ & $1(0.2)$ & $2(2.6)$ & $3(0.4)$ \\
Total & $637(89.1)$ & $78(10.9)$ & $715(100)$ \\
\hline
\end{tabular}

* Artemether-Lumefantrine

$\wedge$ Sulphadoxine-Pyrimethamine

The factors associated with conformity to the 2005 antimalarial treatment policy at bivariate analysis were: doctors compared to nurses $(\mathrm{OR}=107.77$, $\mathrm{CI}=48.21-240.91)$, diagnosis of uncomplicated malaria $(\mathrm{OR}=1.99, \mathrm{CI}=1.22-3.26)$, duration in service less than 6 years $(\mathrm{OR}=10.20, \mathrm{CI}=4.82-$ 21.59), and age of patient 12 years (OR $=3.09$, CI $=1.12-8.55)$. Duration in service was categorized at the median (6 years) while age was categorized at the age of 12 years or less which is usually considered the cut-off age for paediatric patients. The factors that were retained in the multivariate model as independent predictors of conformity to the 2005 antimalarial treatment policy were: duration in service of less than 6 years $(\mathrm{OR}=3.40 . \mathrm{CI}=1.24-9.33)$, doctors compared to nurses $(\mathrm{OR}=97.51, \mathrm{CI}=$
27.29-348.34) and diagnosis of uncomplicated malaria $(\mathrm{OR}=10.13, \mathrm{CI}=3.37-30.42)$. Patient age was retained in the model because of its confounding effect on malaria diagnosis. It changed the odds ratio of malaria diagnosis by more than $10 \%$ when it was omitted from the model. Having stock-outs of antimalarial drugs was not significantly associated with conformity to the antimalarial treatment policy $(\mathrm{OR}=1.01, \mathrm{CI}=0.72-1.44)$. There were 173 patients seen during the period when first line antimalarials were out of stock. The prescribers were informed about the non-availability of the drugs and the patients could be given a prescription to buy the antimalarial drug if they did not get it at the hospital. Results of bivariate and multivariate analysis are summarized in Table 3.

Table 3: Factors associated with conformity to the 2005 malaria treatment policy

\begin{tabular}{|c|c|c|c|c|c|}
\hline Factor & $\begin{array}{l}\text { N }(\%) \\
\text { Conforming }\end{array}$ & $\begin{array}{l}\text { Unadjusted } \\
\text { OR(95\% CI) }\end{array}$ & P-value & $\begin{array}{l}\text { Adjusted } \\
\text { OR(95\%CI) }\end{array}$ & P-value \\
\hline \multicolumn{6}{|l|}{ Sex } \\
\hline Males $(n=250)$ & $235(94.0)$ & $1.91(0.98-3.73)$ & 0.06 & & \\
\hline Females $(n=465)$ & $395(85.0)$ & & & & \\
\hline \multicolumn{6}{|l|}{ Age } \\
\hline$\leq 12$ years $(\mathrm{n}=248)$ & $237(95.6)$ & $3.09(1.12-8.55)$ & 0.03 & $3.36(0.91-12.37)$ & 0.07 \\
\hline$>12$ years $(n=466)$ & $392(84.1)$ & & & & \\
\hline \multicolumn{6}{|l|}{ Malaria diagnosis } \\
\hline Uncomplicated ( $\mathrm{n}=637$ ) & $569(89.3)$ & $1.99(1.22-3.26)$ & 0.006 & $10.13(3.37-30.42)$ & $<0.001$ \\
\hline Complicated $(\mathrm{n}=78)$ & $61(78.2)$ & & & & \\
\hline \multicolumn{6}{|l|}{ Prescriber's profession } \\
\hline $\operatorname{Doctor}(\mathrm{n}=169)$ & $163(96.5)$ & $1.40(0.63-3.10)$ & 0.41 & $1.67(0.59-4.76)$ & 0.033 \\
\hline Clinical officer $(\mathrm{n}=488)$ & $459(94.1)$ & $107.77(48.21-240.91)$ & $<0.001$ & $97.51(27.59-348.34)$ & $<0.001$ \\
\hline Nurses $(\mathrm{n}=58)$ & $8(13.8)$ & & & & \\
\hline \multicolumn{6}{|c|}{ Prescriber's years in service } \\
\hline$<$ Gyearse & $446(96.3)$ & & & & \\
\hline$\geq 6$ years & $184(73.0)$ & & $<0.001$ & $3.40(1.24-9.33)$ & 0.02 \\
\hline \multicolumn{6}{|l|}{ Drugs out of stock } \\
\hline Yes $(n=173)$ & 158(91.3) & $1.01(0.72-1.44)$ & 0.94 & & \\
\hline No $(n=540)$ & $472(87.4)$ & & & & \\
\hline
\end{tabular}




\section{Discussion}

We used data from this rural health facility to evaluate the process of implementation of Uganda's antimalarial treatment policy change. This has been found to be a potentially useful method for monitoring trends in practices. In this study we found that the majority of antimalarial prescriptions in this rural hospital conformed to the 2005 antimalarial treatment policy. The AL was used as first line treatment for uncomplicated malaria in most patients seen and quinine was used as the first line treatment for complicated malaria. Although the level of conformity was high, a small proportion of health service providers were still using CQ, and SP as mono-therapy or in combination with other antimalarial drugs. There were also some prescribers using AL for treating complicated malaria. Even more surprising was the use of SP alone for the treatment of complicated malaria in two of the cases $(2.6 \%)$.

The results show that although most prescribers were conforming to the new antimalarial treatment policy, a considerable number has not adapted to the change $(11.9 \%)$. Some of the treatment decisions taken were not even in agreement with previous treatment guidelines. Despite these exceptions, the proportion of prescriptions conforming to the current Ugandan treatment policy was higher than that found in a study done in Nigeria among medical practitioners ${ }^{18}$. In the latter study despite the change in national treatment guidelines to ACTs, CQ and SP were the most prescribed antimalarials for uncomplicated malaria. The exact explanation for these differences is not clear but may be related to differences in implementation of such policy changes in the two settings. In yet another study by Meremikwu et $\mathrm{al}^{19}$ in South East Nigeria, less than half of the prescriptions from government hospitals (24.4\%) and only $16.7 \%$ of prescriptions from private hospitals consisted of combination therapy. In addition, only $4.6 \%$ of the prescriptions consisted of AL; despite it being the recommended first line treatment for uncomplicated malaria. A review of studies done in Sudan found that the prevalence of inappropriate antimalarial drug prescriptions was at $45 \%{ }^{20}$.

Our study also found that prescribers who had been in service for a shorter time period (less than 6 years) were about 3 times as likely to conform to the policy as those who had been in service longer. This is most likely because the prescribers who had just completed training did so at the time when the drugs recommended in the new policy were available and in use. They may also have been more likely to be aware of the prevailing resistance to previously used antimalarial drugs. Another possible explanation for this is that old habits are hard to change. If health workers have been prescribing a certain drug for patients for a long time, it will be harder for them to change to newer agents. A study on antibiotic prescriptions in Canada found that clinicians who had been in practice longer were more likely to prescribe antibiotics inappropriately ${ }^{21}$.

There was no significant difference between the doctors and clinical officers in conforming to the 2005 antimalarial treatment policy. However, the doctors were 97.5 times more likely to conform to the policy when compared to nurses. Only about 1 in 8 of the prescriptions by nurses conformed to the new treatment policy. In contrast 96 of every 100 prescriptions for doctors conformed to the treatment policy. This is probably because the doctors and clinical officers in Uganda are formally trained to prescribe whereas the nurses only prescribe due to shortage of clinicians but this is not primarily their role. These findings are in agreement with those in another study where higher health worker's cadres were shown to have better prescribing practices ${ }^{22}$. Our findings however contrast with findings from the study by Zurovac et al in Kenya in which nursing aides who are considered less trained adhered much more closely to treatment guidelines than nurses and clinical officers ${ }^{10}$. Given the prevailing health worker shortages at different health facilities, it would therefore be critical to ensure that all cadres of staff are trained on such new treatment policies to ensure that these skills are provided to all members of the team who may take key prescribing responsibilities.

Prescribers were also more likely to conform to the antimalarial treatment policy when the diagnosis made was uncomplicated malaria. This may be attributed to the fact the majority of training programmes particularly emphasize the management of uncomplicated malaria with little emphasis on management of severe malaria.

Prescriptions for younger children were more likely to conform to antimalarial policy. This is possibly because illness in this group is perceived to be more serious than in adults and therefore more care may be taken. In addition, since this group bears the greatest brunt of malaria illness it can be assumed that they present more frequently to the health units hence health workers may have more experience in 
handling them. Age has also been associated with prescribing practices in Kenya and Ghana ${ }^{10,23}$.

Having stock-outs of antimalarial drugs was not associated with conformity to the malaria treatment policy. This is possibly because the health workers prescribe drugs for patients regardless of what is in stock, since patients can be directed to buy the medicines outside the health facility.

Despite the fact that the majority of prescriptions conformed to the antimalarial treatment policy, it is of great concern that both AL and quinine were prescribed concurrently for some patients. Such misuse of available antimalarial drugs may cause unnecessary increases in drug costs as well as increasing the risk of adverse events.

Our study had some limitations; correctness of the prescribed doses was not ascertained as information on dosage was missing from the register for many of the patients. In addition, weights were taken for only 9 in every 100 patients. This implies that adequacy of weight-based dosage could not be assessed. It would have been important to assess whether appropriate dosing instructions were given to the patient but since we were using records this could not be done.

\section{Conclusion}

The findings from this study indicate that although the majority of health workers conformed to the antimalarial treatment policy, a few of the prescriptions were contrary to the new treatment guidelines. Given the fact that the implementation of a national drug policy is usually faced with several constraints, it is important to ensure that adequate training is done, with provision of relevant information, as well as effective communication with health workers to promote behavior change and effective uptake of policy changes. These efforts should especially address the specific needs and challenges of different cadres of prescribers as well as persons that have been in service for longer periods, who have been using older treatment guidelines. There is also need to conduct further studies to assess other aspects of the treatment like dosage and proper drug administration.

\section{Acknowledgements}

We are grateful to the staff and administration of Kalisizo hospital for their support and cooperation. We thank all members of the study team, Sr. Nakimbugwe and Sr. Nagirinya for working tirelessly to get this work accomplished; and Levi Mugenyi for data analysis.

\section{References}

1. Malaria - WHO Fact sheet No 94 [http:// www.who.int/mediacentre/factsheets/fs094/ en/]

2. Saving lives in Africa - Malaria in Uganda [http:/ /www.who.pmi.gov/countries.uganda]

3. White NJ, Nosten F, Looareesuwan S, Watkins WM, Marsh K, Snow RW: Averting a malaria disaster. Lancet 1999; 353:1965-1967.

4. Bloland PB: Drug resistance in Malaria. World health Organization Bulletin 2001; 4.

5. Trape JF: The public health impact of Chloroquine resistance in Africa. American Journal of Tropical Medicine and Hygiene 2001; 64(1-2Supplement):1217.

6. Kamya MR, Bakyaita NN, Talisuna A, Were WM, Staedke SG: Increasing antimalarial drug resistance in Uganda and revision of the National Drug Policy. Tropical Medicine \& International Health 2002;7(12):1031-1041.

7. Uganda Ministry of Health (MOH): National Treatment guidelines, 2 edn. Kampala; 2003.

8. Zurovac D, Ndhlovu M, Rowe AK, Hamer DH, Thea DM, Snow RW: Treatment of Pediatric malaria during a period of drug transition to Artemether-Lumefantrine in Zambia: Crosssectional study. Br Med J 2005; 331:706-707.

9. Williams HA, Durrheim D, Shretta R: The process of changing natinal malaria treatment policy: lessons from country level studies. Health policy and planning 2004; 19(6):356-370.

10. Zurovac D, Rowe AK, Ochola S, Noor AM, Midia B, English E, Snow RW: Predictors of the quality of health worker treatment practices for uncomplicated malaria at government health facilities in Kenya. International Journal of epidemiology 2004; 33(5):1080-1091.

11. WHO: Drug Supply Management, 2nd edn: WHO; 2001.

12. Zurovac D, Tibenderana JK, Nankabirwa J, Ssekitooleko J, Njogu JN, Rwakimari JB, Meek S, Talisuna A, Snow RW: Malaria casemanagement under artemether-lumefantrine treatment policy in Uganda. Malar J 2008; 7:181.

13. Kyabayinze D, Asiimwe C, Nakanjako D, Nabakooza J, Counihan H, Tibenderana J. Use of RDTs to improve malaria diagnosis and fever case management at primary health care facilities in Uganda. Malaria Journal. 2010;9(1):200. 
14. Uganda Bureau of Statistics (UBOS), Macro International Inc. Uganda Demographic and Health Survey 2006. Calverton: UBOS and Macro International Inc2007.

15. Chadha V: Sample size determination in health studies. NTI Bulletin 2006; 42(3\&4):55-62.

16. Cadieux G TR, Dauphinee D, Libman M.: Predictors of inappropriate antibiotic prescribing among primary care physicians. CMAJ 2007; 177(8): 877-883.

17. Vittinghoff Eric GVD, Shiboski C Stephen, McCulloch E Charles Regression methods in Biostatistics: Linear, Logistic, Survival and Repeated Measures Models.Springer; 2005.

18. Oshikoya KA: Antimalarial Prescriptions for Children Presenting with Uncomplicated Malaria to a Teaching. Hospital in Nigeria after the Change of National Guidelines for Malaria Treatment. . World Journal of Medical Sciences 2007; 2(1): 49-53.

19. Meremikwu M, Okomo U, Nwachukwu C, Oyo-Ita A, Eke-Njoku J, Okebe J, et al. Antimalarial drug prescribing practice in private and public health facilities in South-east Nigeria: a descriptive study. Malaria Journal. 2007;6(1):55.

20. Awad AI, Ball DE, Eltayeb BI: Improving rational drug use in Africa: the example of Sudan. East African Mediterranean Journal 2007; 13(5):1202-11

21. Cadieux G, Tamblyn R, Dauphinee D, Libman M: Predictors of inappropriate antibiotic prescribing among primary care physicians. CMAJ 2007; 177(8): 877-883.

22. Zurovac D, Njogu J, Akhwale WS, Hamer DH, Snow RW: Translation of ArtemetherLumefantrine treatment policy into paediatric clinical practice: an early experience from Kenya. Tropical Medicine \& International Health 2008; 13(1): 99-107.

23. Dodoo A, Fogg C, Asiimwe A, Nartey E, Kodua A, Tenkorang $\mathrm{O}$, et al. Pattern of drug utilization for treatment of uncomplicated malaria in urban Ghana following national treatment policy change to artemisinin-combination therapy. Malaria Journal. 2009;8(1):2. 